ENCYCLOPEDEE Encyclopédie berbère

BERBERE

21 | 1999

21 | Gland - Hadjarien

\title{
Haches polies
}

G. Souville

\section{(2) OpenEdition}

Journals

Édition électronique

URL : http://journals.openedition.org/encyclopedieberbere/1843

DOI : $10.4000 /$ encyclopedieberbere. 1843

ISSN : 2262-7197

\section{Éditeur}

Peeters Publishers

\section{Édition imprimée}

Date de publication : 1 septembre 1999

Pagination : 3272-3275

ISBN : 2-7449-0097-4

ISSN : $1015-7344$

\section{Référence électronique}

G. Souville, "Haches polies », Encyclopédie berbère [En ligne], 21 | 1999, document H04, mis en ligne le

01 juin 2011, consulté le 25 septembre 2020. URL : http://journals.openedition.org/

encyclopedieberbere/1843; DOI : https://doi.org/10.4000/encyclopedieberbere.1843

Ce document a été généré automatiquement le 25 septembre 2020

(C) Tous droits réservés 


\section{Haches polies}

\section{G. Souville}

1 Les haches polies déjà connues de Pline (Hist. nat., XXXVII, 48-51) sont certainement les outils préhistoriques les plus anciennement signalés, sans doute en raison de leur matière première, de leur couleur et de leur sensation au toucher. Elles ont d'ailleurs été parfois considérées comme des talismans.

2 On admettait généralement que ces haches étaient rares au Maghreb. Cela est partiellement vrai pour les découvertes in situ mais on les trouve en grande abondance dans les gisements de surface. Près de 900 haches viennent de la région de Mazagan au Maroc (Souville G., Atlas préhistorique du Maroc, 1, Le Maroc atlantique, Paris, 1973, p. 279-287), plus de 600 des différents sites de l'oued Beth (ibid., p. 149-163) mais aussi une quarantaine à Volubilis (ibid., p.138-141) et à Bab Merzouka (Souville G., «L'industrie préhistorique de Bab Merzouka (Maroc)», in L'Homme méditerranéen. Mélanges offerts à G. Camps, 1995, p. 93-100) et une trentaine à Taforalt, toujours au Maroc. On doit en signaler également 70 dans la région de Tiaret (Cadenat P., « Notes de préhistoire tiarétienne, 1, Les haches, herminettes et pilons", Libyca, t.12, 1964, p. 181-224) et une centaine à Brezina, dans le sud oranais (Souville G., «Précisions sur la classification... », op. 1. infra, p. 382). 
Hache triangulaire et épaisse au biseau symétrique et au tranchant curviligne ; entièrement bouchardée (oued Beth, Maroc).

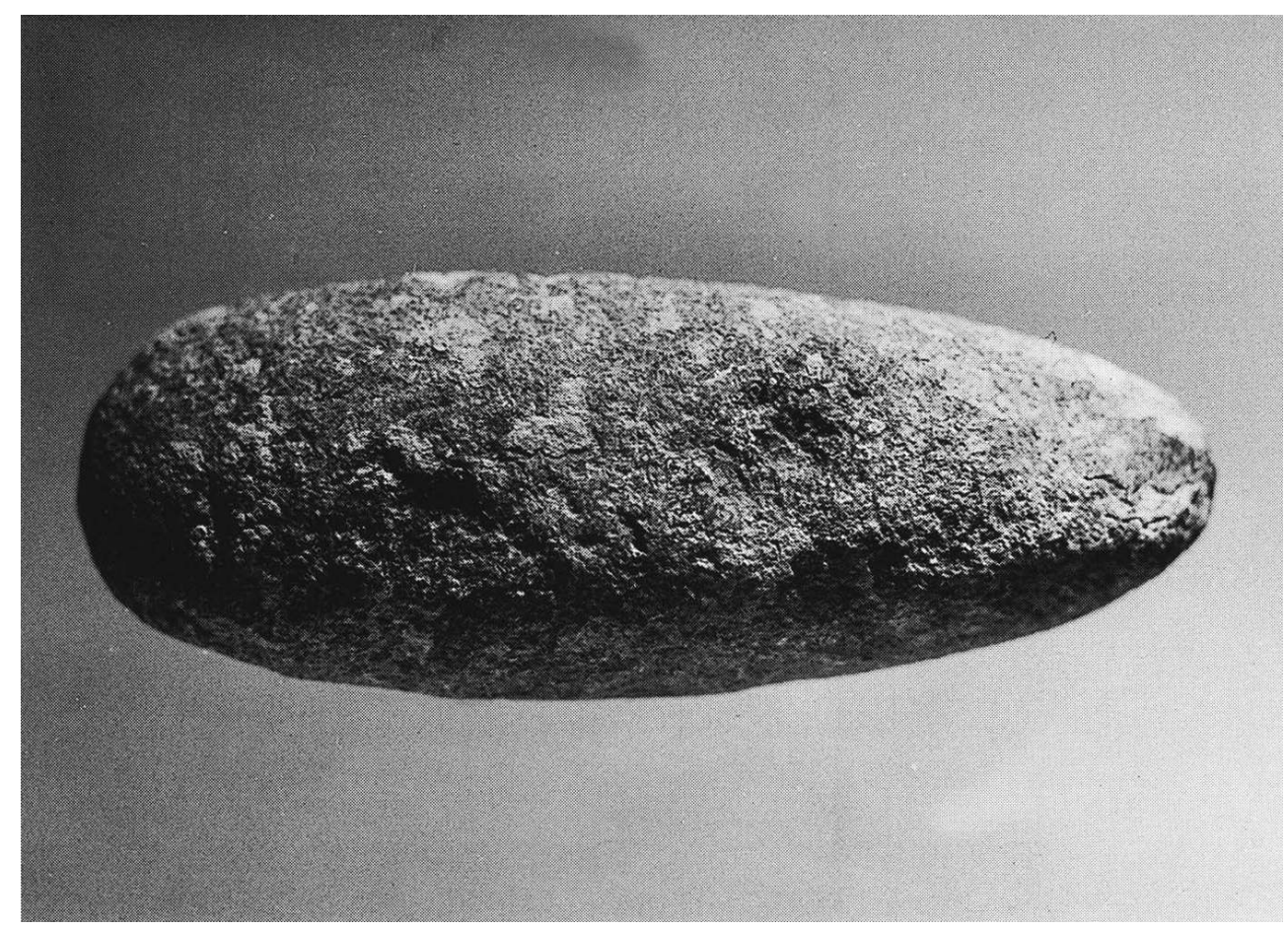

Hache trapézoïdale épaisse ; entièrement polie (Achakar, Maroc).

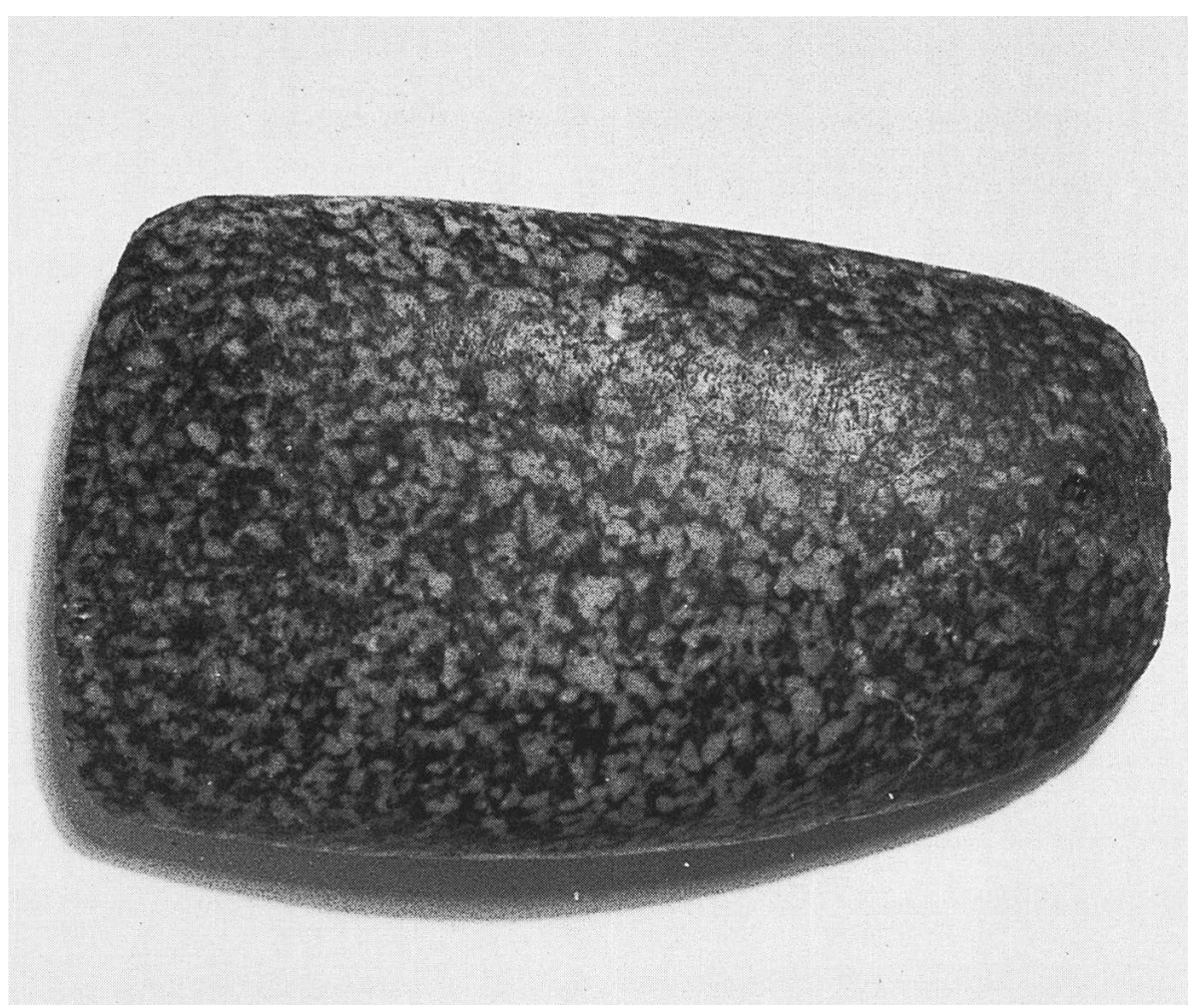


Hache en boudin ; biseau symétrique ; tranchant curviligne ; partie proche du tranchant polie ; le reste bouchardé (Mazagan, Maroc).

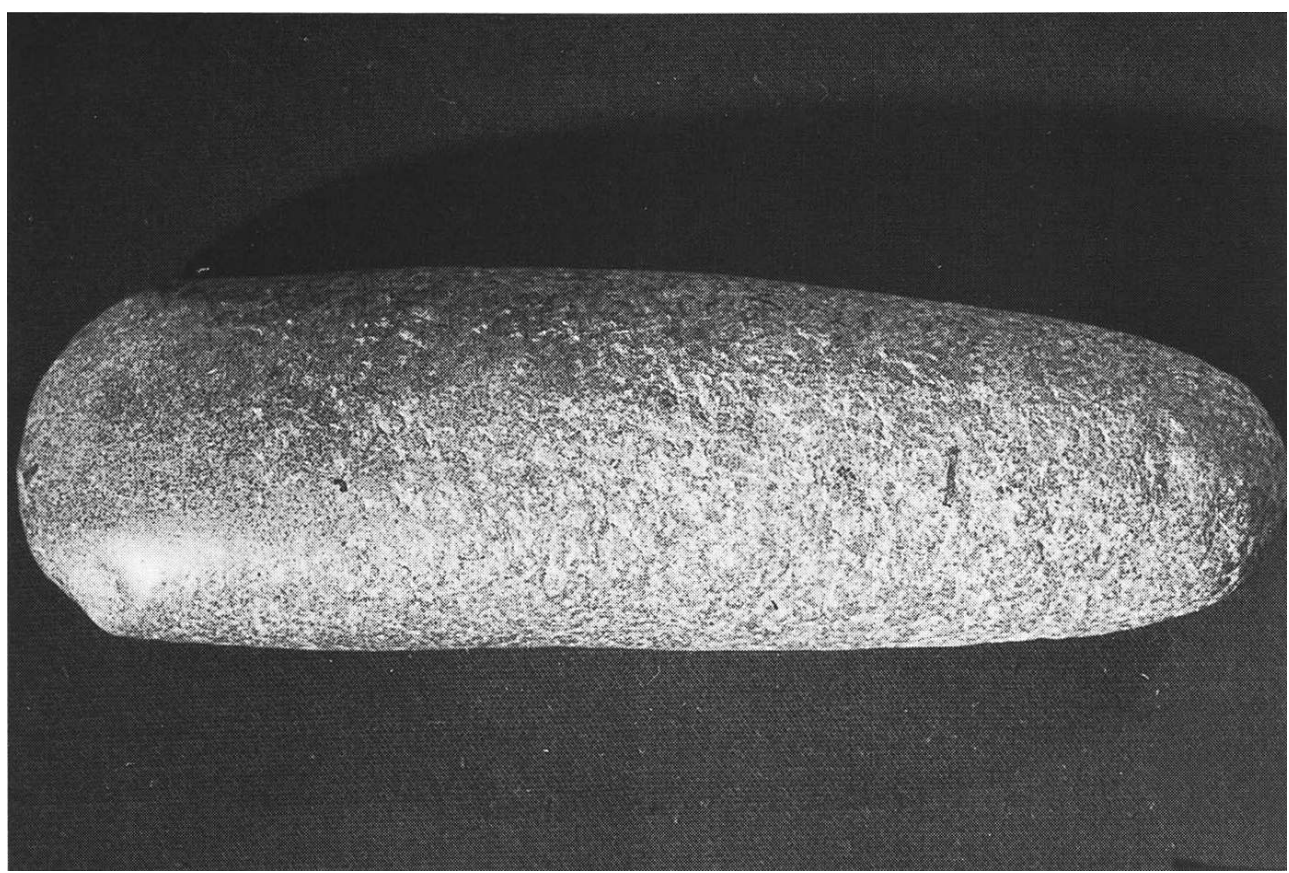

Haches polies de la région de Tiaret, photo $\mathrm{P}$. Cadenat.

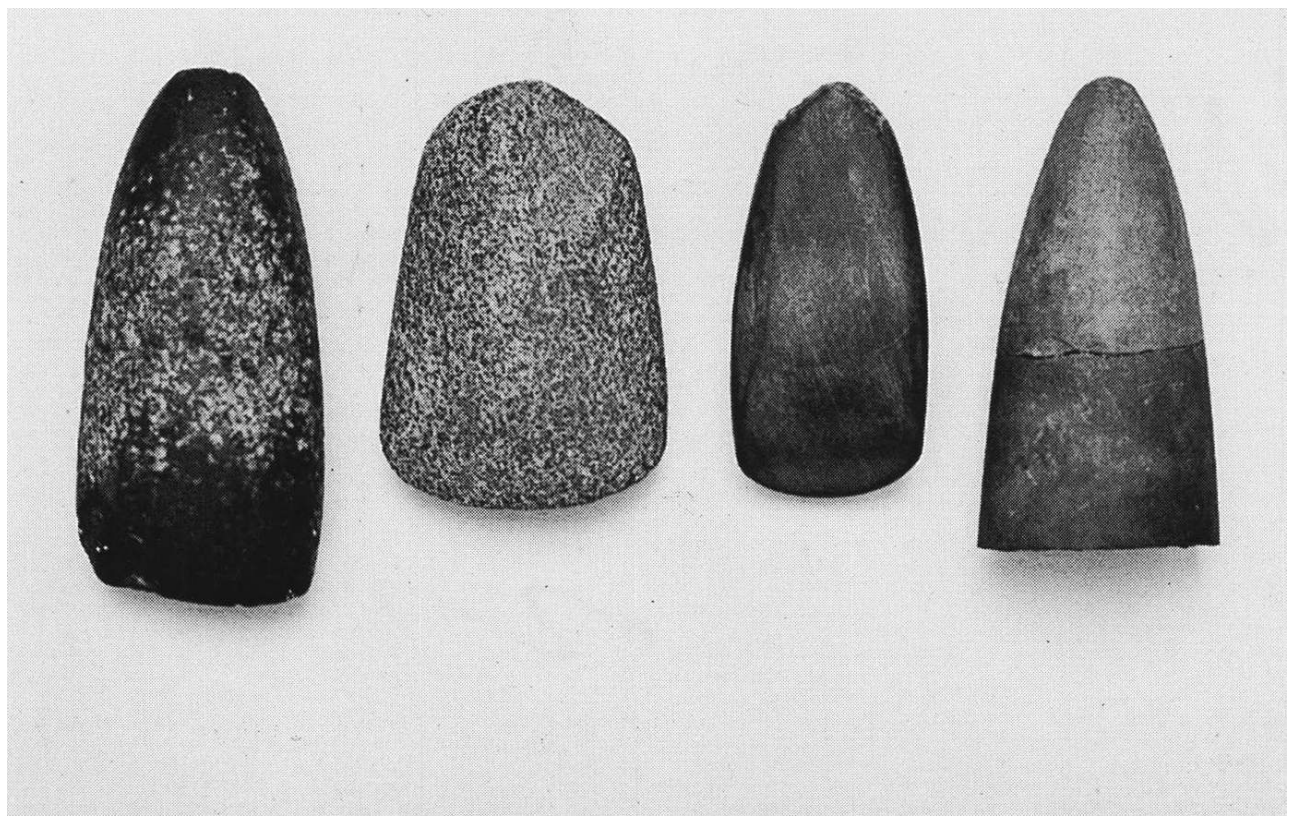

3 Les formes sont variées : triangulaires, rectangulaires ou trapézoïdales, rarement en boudin. Les haches sont épaisses ou plates; le tranchant est symétrique ou asymétrique, avec le cas particulier des erminettes, rectiligne ou curviligne, parfois épais (jusqu'à un cm). 
Hache de l'oued Sly, Algérie, la plus grande des haches trouvées en Afrique du nord (longueur : 34 cm) (photo M. Bovis).

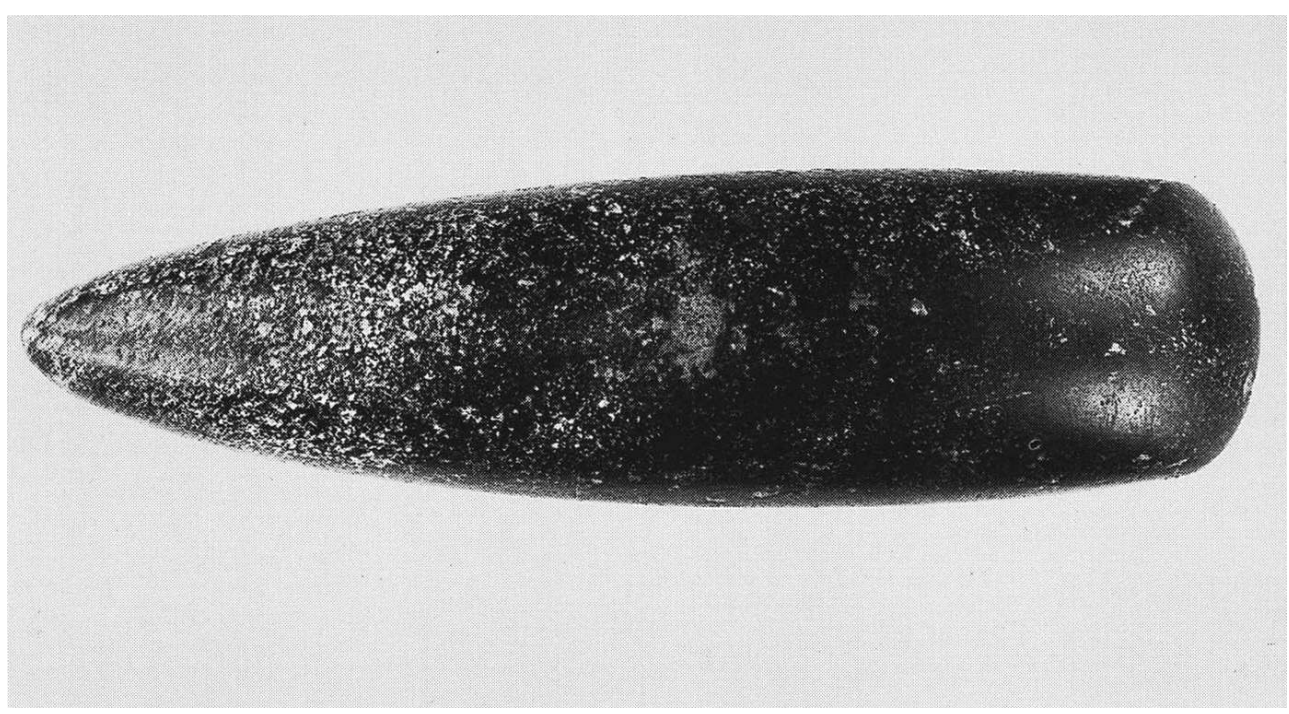

4 La forme dominante est triangulaire, plus souvent épaisse que plate, à la section ovalaire, avec un tranchant souvent curviligne.

5 Ces haches ne sont que très rarement entièrement polies. Elles peuvent être complètement bouchardées et finement piquetées sur toute leur surface. Mais en général le tranchant et les parties voisines sont soigneusement polies et le reste bouchardé. Certes le poli a pu être accentué par l'usage et surtout la nécessité d'aiguiser le tranchant. Mais souvent le poli est antérieur au bouchardage comme si l'ouvrier avait commencé par la partie utile de l'objet, terminant ensuite par bouchardage.

6 Il est difficile d'avoir une idée précise sur les modes d'utilisation de ces objets. La plupart étaient probablement emmanchés et pouvaient servir de haches voire de massues. Certaines, très petites, étaient sans doute votives; elles pouvaient aussi être des jouets d'enfant. Quant aux erminettes, elles ont pu servir de houes, comme les " haches taillées".

7 Quelle est leur position chronologique ? Certaines ont été trouvées en place dans un site néolithique comme à Damous el Ahmar en Algérie orientale (Roubet $\mathrm{C}$, Le gisement de Damous el Ahmar et sa place dans le Néolithique de tradition capsienne, Paris, 1968, p. 46-52). Il existe quelques haches aux bords concaves et au tranchant débordant avec deux épaulements qui rappellent les haches en métal. Mais la plupart d'entre elles ont été trouvées en surface, mêlées à une industrie néolithique mais aussi épipaléolithique voire plus ancienne. Elles ont pu perdurer après le Néolithique jusqu'au chalcolithique et même au delà, comme d'autres objets en pierre polie du Maghreb (pierres à rainure et à cupules, pièces à gorge). 


\section{BIBLIOGRAPHIE}

SOUVILLE G, « Note typologique sur des haches polies du Maghreb », Libyca, Anthr.-Préhist.-Ethn., t. 16,1968 , p. $145-151$.

Id., « Précisions sur la classification des haches polies du Maghreb », Miscelánea arqueológica, Barcelona, 1974, t. 2, p. 381-387.

INDEX

Mots-clés : Arme, Préhistoire 